\title{
Lean mass, grip strength and risk of type 2 diabetes: a bi-directional Mendelian randomisation study
}

\author{
Chris Ho Ching Yeung ${ }^{1}$ - Shiu Lun Au Yeung ${ }^{1}$ - Shirley Siu Ming Fong ${ }^{1} \cdot$ C. Mary Schooling ${ }^{1,2}$
}

Received: 27 June 2018 / Accepted: 11 January 2019 / Published online: 23 February 2019

(C) Springer-Verlag GmbH Germany, part of Springer Nature 2019

\begin{abstract}
Aims/hypothesis Muscle mass and strength may protect against type 2 diabetes as a sink for glucose disposal. In randomised controlled trials, resistance training improves glucose metabolism in people with the metabolic syndrome. Whether increasing muscle mass and strength protects against diabetes in the general population is unknown. We assessed the effect of markers of muscle mass and strength on diabetes and glycaemic traits using bi-directional Mendelian randomisation.

Methods Inverse variance weighting estimates were obtained by applying genetic variants that predict male lean mass, female lean mass and grip strength, obtained from the UK Biobank GWAS, to the largest available case-control study of diabetes (DIAbetes Genetics Replication And Meta-analysis [DIAGRAM]; $n=74,124$ cases and 824,006 controls) and to a study of glycaemic traits (Meta-Analyses of Glucose and Insulin-related traits Consortium [MAGIC]). Conversely, we also applied genetic variants that predict diabetes, $\mathrm{HbA}_{1 \mathrm{c}}$, fasting glucose, fasting insulin and HOMA-B to UK Biobank summary statistics for genetic association with lean mass and grip strength. As sensitivity analyses we used weighted median, Mendelian randomisation (MR)-Egger and Mendelian Randomization Pleiotropy RESidual Sum and Outlier (MR-PRESSO) and removed pleiotropic SNPs.

Results Grip strength was not significantly associated with diabetes using inverse variance weighting (OR 0.72 per SD increase in grip strength, 95\% CI 0.51, 1.01, $p=0.06$ ) and including pleiotropic SNPs but was significantly associated with diabetes using MR-PRESSO (OR 0.77, 95\% CI 0.62, 0.95, $p=0.02$ ) after removing pleiotropic SNPs. Female lean mass was significantly associated with diabetes (OR $0.91,95 \%$ CI $0.84,0.99, p=0.02$ ) while male lean mass was not significant but directionally similar (OR 0.94, 95\% CI 0.88, 1.01, $p=0.09$ ). Conversely, diabetes was inversely and significantly associated with male lean mass ( $\beta$ -0.02 SD change in lean mass, $95 \% \mathrm{CI}-0.04,-0.00, p=0.04)$ and grip strength $(\beta-0.01,95 \% \mathrm{CI}-0.02,-0.00, p=0.01)$.

Conclusions/interpretation Increased muscle mass and strength may be related to lower diabetes risk. Diabetes may also be associated with grip strength and lean mass. Muscle strength could warrant further investigation as a possible target of intervention for diabetes prevention.
\end{abstract}

Keywords Body composition · Diabetes mellitus · Grip strength · Hand strength · Lean mass · Mendelian randomisation · Muscle · Type 2 diabetes

Electronic supplementary material The online version of this article (https://doi.org/10.1007/s00125-019-4826-0) contains peer-reviewed but unedited supplementary material, which is available to authorised users.

C. Mary Schooling

cms1@hku.hk

1 School of Public Health, Li Ka Shing Faculty of Medicine, The University of Hong Kong, 7 Sassoon Road, Pokfulam, Hong Kong SAR, China

2 Graduate School of Public Health and Health Policy, City University of New York, New York, USA

\author{
Abbreviations \\ DIAGRAM DIAbetes Genetics Replication And Meta- \\ analysis \\ GWAS Genome-wide association study \\ InSIDE Instrument Strength Independent of Direct \\ Effect \\ MAGIC Meta-Analyses of Glucose and Insulin-relat- \\ ed traits Consortium \\ MR-Egger Mendelian randomisation-Egger \\ MR-PRESSO Mendelian Randomization Pleiotropy \\ RESidual Sum and Outlier
}




\section{Research in context}

\section{What is already known about this subject?}

- Resistance training improves glucose metabolism in people with the metabolic syndrome but the protective effect of muscle mass and strength on diabetes in the general population is unknown

\section{What is the key question?}

- What are the effects of markers of muscle mass and strength on diabetes and glycaemic traits in the general population?

What are the new findings?

- In a bi-directional Mendelian randomisation study, higher grip strength was significantly associated with a decreased risk of diabetes

- Higher female lean mass was significantly associated with decreased risk of diabetes, while the association for male lean mass was not significant but was directionally similar

- Conversely, diabetes was significantly associated with lower male lean mass and lower grip strength

\section{How might this impact on clinical practice in the foreseeable future?}

- Muscle strength could warrant further investigation as a possible target of intervention for improving glycaemic traits and preventing diabetes

\section{Introduction}

Type 2 diabetes is increasingly common globally, particularly in Asia, where rates of diabetes are high even in populations with comparatively low rates of obesity [1], suggesting the role of factors beyond adiposity. Low birthweight is a wellestablished cause of diabetes [2], but is difficult to modify, meaning that the consequences of low birthweight might be more effective and timely targets of intervention. Low birthweight is also associated with low muscle mass [3], which is modifiable and could mediate the effect of birthweight on diabetes. Muscle may act as a sink for glucose disposal [4], and muscle mass is inversely associated with diabetes observationally [5]. However, these observational studies could be confounded by many factors including health status, exercise habits and height, resulting in spurious associations $[4,5]$. Randomised controlled trials have shown some benefits of resistance training for glucose metabolism in people with the metabolic syndrome [6]. However, these randomised controlled trials are primarily designed to investigate the therapeutic effects of resistance training in people with poor glucose metabolism or diabetes, rather than to assess whether muscle mass and strength are protective against diabetes in the general population.

In this situation, Mendelian randomisation studies may provide evidence to guide the search for effective interventions for preventing diabetes in the general population. Mendelian randomisation, as an instrumental variable analysis with genetic instruments, uses predicted levels of exposure experienced by an individual [7]. Given that genetic makeup is randomly allocated at conception, genotype is likely to be independent of the factors that may confound observational studies and can distinguish symptoms from causes (i.e. avoid reverse causality) [8]. Mendelian randomisation studies could be thought of as comparable with randomised controlled trials with random assortment of genotypes acting as a randomisation process to allocate individuals to different levels of exposure [8]. Specifically, we used a two-sample Mendelian randomisation design obtaining summary statistics for SNPs predicting exposure and applying them to summary statistics from a GWAS of the outcomes [9]. The use of a two-sample design has several advantages, such as limiting bias due to the 'winner's curse' [10]. The use of summary statistics from consortia also has the advantage of increased statistical power [9]. No association of grip strength with diabetes has been reported from a somewhat limited Mendelian randomisation study only using two SNPs for grip strength [11]. To our knowledge, no other Mendelian randomisation study has been conducted on this topic.

In order to test the hypothesis that there is a causal role of muscle mass and strength in diabetes and glycaemic traits, as well as to assess any potential reverse causality, we used a bidirectional two-sample Mendelian randomisation study design. We assessed whether genetically higher lean mass and grip strength (as proxies of muscle mass and muscle strength) were associated with diabetes and glycaemic traits. Conversely we also assessed whether genetically higher risk of diabetes or poor glycaemic traits were associated with lean mass and grip strength. 


\section{Methods}

\section{Influence of genetically predicted lean mass and grip strength on risk of diabetes and glycaemic traits}

Whole body lean mass and grip strength Genetic predictors (SNPs) of whole body lean mass (fat-free mass) for men and women, as well as grip strength (right hand for both sexes) were obtained from the most up-to-date GWAS of the UK Biobank, which included 361,194 participants recruited in the UK [12]. Briefly, the UK Biobank recruited over 500,000 participants aged 40-69 years in 2006-2010, who provided comprehensive information, underwent extensive tests and provided biological specimens $[12,13]$. Lean mass was measured using bioelectrical impedance analysis. Grip strength was measured isometrically using a calibrated hydraulic hand dynamometer adjusted for hand size [14]. Sexspecific genetic associations were adjusted for the first 20 principal components, age and age $^{2}$, while sex, sex $\times$ age and sex $\times \mathrm{age}^{2}$ were additionally adjusted for in analyses of both sexes. Sample and variant quality controls, such as restriction to white British genetic ancestry, genetic variant information metric (INFO score) $>0.8$ and minor allele frequency $>0.0001$ were also applied [15].

Lean mass represents lipid-free soft tissue including muscle, body water, protein, glycerol and soft tissue mineral mass [16], which is considered to be a valid measure of skeletal muscle mass [17]. For grip strength, we used absolute rather than relative grip strength (absolute grip strength/weight) as a proxy for muscle strength, because absolute grip strength may have a higher correlation with general muscle strength than relative grip strength $[18,19]$.

Diabetes Genetic associations with diabetes were obtained from the DIAMANTE (European) meta-analysis of type 2 diabetes from the DIAbetes Genetics Replication And Metaanalysis (DIAGRAM) consortium [20]. The study is a metaanalysis of diabetes case-control studies $(n=74,124$ cases and 824,006 controls) of people of European ancestry from 32 studies $(49.6 \%$ cases and $48.0 \%$ controls were women; mean age cases 55.2 years, controls 52.7 years) adjusted for study specific covariates and principal components for ancestry [20].

Glycaemic traits Genetic associations with glycaemic traits were obtained from the Meta-Analyses of Glucose and Insulin-related traits Consortium (MAGIC) [21-23]. We used a meta-analysis of GWAS results including $\mathrm{HbA}_{1 \mathrm{c}}$ (in $\%$ units) for 123,665 people (European ancestry only, 61.4\% women), fasting glucose for 58,074 people $(51.3 \%$ women, mean age men 55.5 years; women 54.1 years), fasting insulin for 51,750 people (49.7\% women, mean age men 56.9 years, women 55.3 years) and indices of insulin resistance (HOMA-IR, 55.5\% women, mean age men 51.4 years; women 50.9 years) and beta cell function
(HOMA-B, 55.6\% women, mean age men 51.3 years; women 50.8 years) for up to 46,186 people [21, 22]. All studies were adjusted for age, sex and study specific covariates [21-23]. The GWAS of $\mathrm{HbA}_{1 \mathrm{c}}$ also accounted for population structure using genomic control [21]. Paired fasting glucose and insulin measures were used to calculate HOMA-B and HOMA-IR with the homeostasis model assessment [24].

\section{Influence of genetically predicted diabetes risk and glycaemic traits on lean mass and grip strength}

Diabetes and glycaemic traits Genetic predictors of diabetes, $\mathrm{HbA}_{1 \mathrm{c}}$ and HOMA-B were obtained from DIAGRAM and MAGIC. Significant SNPs for diabetes were extracted from the original summary statistics provided by DIAGRAM [20]. The SNPs for $\mathrm{HbA}_{1 \mathrm{c}}$ (European ancestry only) were obtained from published supplementary files [21]. For fasting glucose and fasting insulin, we obtained genetic associations reaching genome-wide significance $\left(p<5 \times 10^{-8}\right)$ from the GWAS by Scott et al of up to 133,010 individuals (fasting glucose: $51.6 \%$ men, mean age men 51.35 years; women 50.0 years; fasting insulin: $52.7 \%$ men, mean age men 50.7 years; women 49.2 years) adjusted for age, study site and geographical covariates, with genomic control [25].

Lean mass and grip strength Genetic associations with whole body lean mass (fat-free mass) and grip strength were obtained from genetic summary statistics of the UK Biobank [12, 13].

\section{Selection of genetic predictors}

We used all SNPs that independently (linkage disequilibrium $\left.r^{2}<0.01\right)$ and strongly, at genome-wide significance $(p<5 \times$ $\left.10^{-8}\right)$, predicted an exposure. For whole body lean mass, grip strength and diabetes, we used a lower cut-off ( $p$ value $=0.05$ / number of SNPs examined) for genome-wide significance to avoid false positives given the large number of SNPs in these GWASs. We computed the $F$ statistic for SNPs on exposures to assess the overall and individual instrument strength [26-28]. If the variance explained by the SNPs $\left(r^{2}\right)$ was not available, we estimated the pooled variance for calculating the $r^{2}$, as given in the electronic supplementary material (ESM) Methods. To assess the exclusion restriction assumption in Mendelian randomisation, we searched the curated SNP-tophenotype databases PhenoScanner (Version 2; http://www. phenoscanner.medschl.cam.ac.uk/) and the GWAS Catalog (https://www.ebi.ac.uk/gwas) [29, 30], for other genomewide significant $\left(p<5 \times 10^{-8}\right)$ phenotypes related to the chosen SNPs and their correlated SNPs, which may affect the outcomes via paths other than the exposures of interest (i.e. pleiotropic SNPs). We also used Mendelian Randomization Pleiotropy RESidual Sum and Outlier (MR-PRESSO) to identify and remove pleiotropic SNPs [31]. 


\section{Statistical analysis}

In the main analysis, we present estimates with $95 \% \mathrm{CI}$ and $p$ values from inverse variance weighting with multiplicative random effects, which essentially combines the SNP-specific Wald estimates assuming balanced pleiotropy. Wald estimates were calculated as the SNP-outcome estimate divided by the SNP-exposure estimate. We aligned palindromic SNPs (allele pairs coded as $\mathrm{A} / \mathrm{T}$ or $\mathrm{C} / \mathrm{G}$ ) on allele and effect allele frequency. For fasting glucose, fasting insulin, HOMA-B and HOMA-IR, we excluded palindromic SNPs before removing SNPs in linkage disequilibrium because effect allele frequency is not given so they cannot be unequivocally aligned with outcome SNPs. Heterogeneity of the Wald estimates was assessed using $I^{2}$, where a high $I^{2}$ may indicate the presence of invalid instruments. Given that it is difficult to verify the exclusion restriction assumption, we conducted several sensitivity analyses that rely on different assumptions for valid estimation. A $p$ value $<0.05$ was considered to be statistically significant. No correction for multiple comparison was performed as the primary analysis essentially focused on testing the one hypothesis that muscle mass and strength are associated with risk of diabetes. Power calculations were performed [32]. We assessed whether association differed by sex from the differences in slope [33]. There were 41 tests of sex differences altogether and therefore we applied a Bonferroni corrected significance level (0.05/ $41=0.001)$ to account for multiple comparisons.

All analyses were performed using R Version 3.4.2 with the R package (TwosampleMR) [34, 35].

\section{Sensitivity analysis}

Weighted median We present weighted median estimates which are valid estimates when at least $50 \%$ of information is derived from valid SNPs [36].

MR-Egger Mendelian randomisation (MR)-Egger regression provides valid estimates even when all the SNPs are invalid, provided that the Instrument Strength Independent of Direct Effect (InSIDE) assumption holds [37]. An MR-Egger intercept where the $p<0.05$ indicates the presence of directional pleiotropy. However, MR-Egger has lower statistical power compared with inverse variance weighting and hence we focused more on the consistency of the estimate direction between MR-Egger and inverse variance weighting.

MR-PRESSO MR-PRESSO detects horizontal pleiotropy by assessing outliers among the included SNPs contributing to the Mendelian randomisation estimate. This method assumes that at least $50 \%$ of the SNPs are valid SNPs, have balanced pleiotropy and the InSIDE assumption holds. MR-PRESSO also identifies the outlier SNPs and provides adjusted estimates [31]. We used this method as a statistical procedure to locate and remove possible pleiotropic SNPs.

Removal of pleiotropic SNPs We repeated the analyses after excluding potentially pleiotropic SNPs. Considering the close relation between lean mass and grip strength and the difficulty of unequivocally identifying pleiotropic SNPs, we used two different approaches: strict and lenient. Briefly, in the strict approach we removed all SNPs related to any aspect of body composition except the relevant exposure, while in the lenient approach we removed all pleiotropic SNPs including those related to fat mass, on the assumption that fat causes diabetes [38], but not the SNPs related to body composition, which may be associated with lean mass or grip strength, e.g. height and weight. The lenient approach has the advantages of removing key pleiotropic SNPs while preserving statistical power but risks including some pleiotropic SNPs. The strict approach removes all invalid SNPs but possibly also some valid SNPs.

\section{Ethics approval}

We used publicly available summary data where no ethical approval is required.

\section{Results}

\section{Influence of genetically predicted lean mass and grip strength on risk of diabetes and glycaemic traits}

We obtained 313 SNPs for male whole body lean mass, 311 for female whole body lean mass and 130 for grip strength after removing correlated SNPs. The $F$ statistics for individual SNPs were all larger than 10 , with overall $F$ statistics and variance explained by SNPs of 117 and $8 \%$ for male whole body lean mass, 101.9 and $6.9 \%$ for female whole body lean mass and 26.4 and $0.7 \%$ for grip strength [13]. Attributes of the SNPs are shown in ESM Tables 1-3. ESM Table 4 shows the phenotypes related to the potentially pleiotropic SNPs using the strict and lenient definitions.

Table 1 shows no association of male whole body lean mass with any outcome robust to sensitivity analysis based on 180 SNPs using the lenient definition of pleiotropic SNPs or on eight SNPs using the strict definition, although it is associated with fasting glucose using inverse variance weighting and the lenient approach. Female lean mass was inversely and significantly associated with diabetes using the lenient pleiotropy definition with consistent estimates in all sensitivity analyses but was not significantly associated with any outcome using the strict pleiotropy definition SNPs. No significant sexspecific effect was found after correcting for multiple comparisons as shown in Table 2. Grip strength was inversely and significantly associated with diabetes using the lenient 


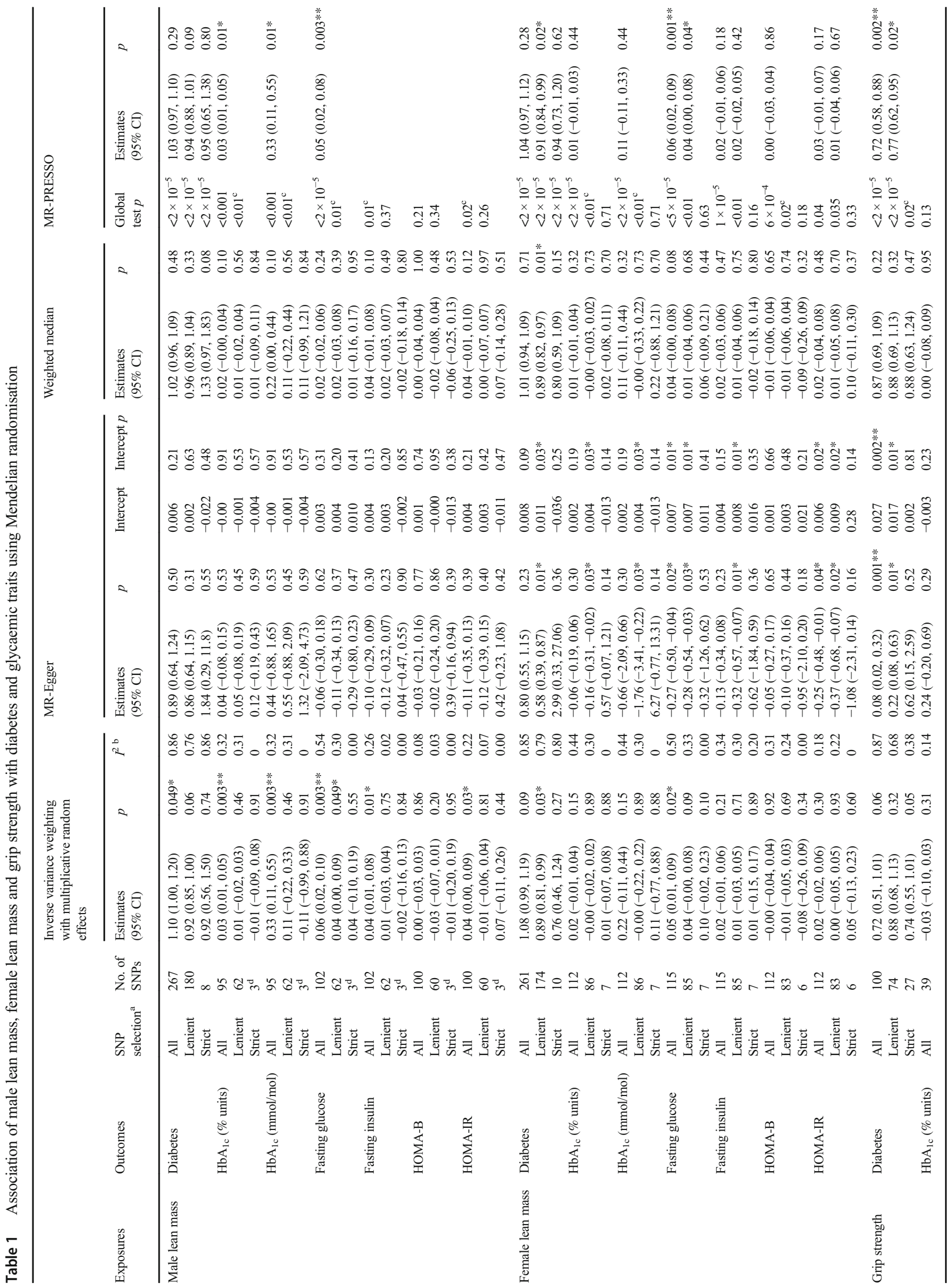




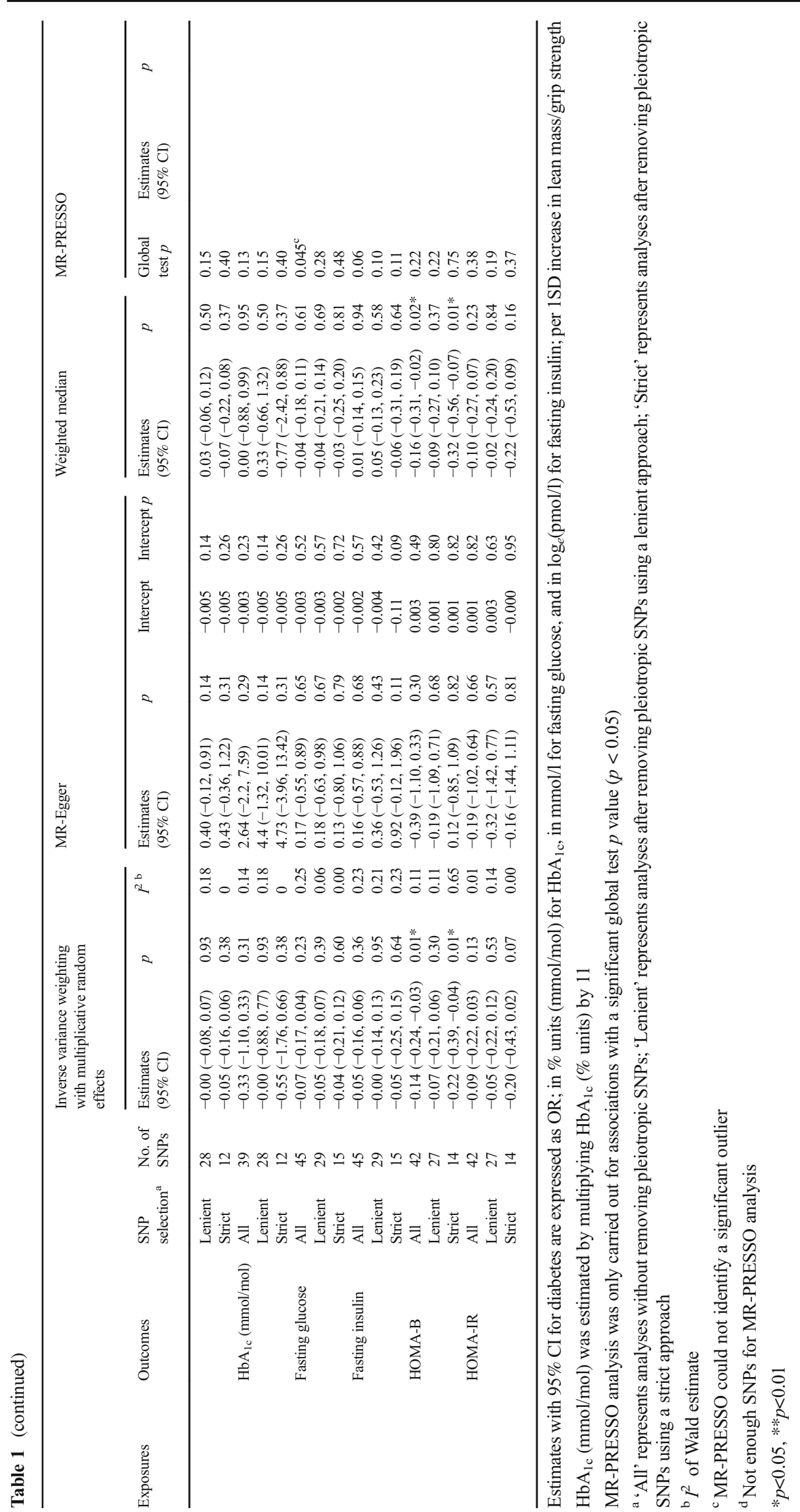


Table 2 Assessment of differences by sex, $\mathrm{p}$ values for tests of interaction

\begin{tabular}{|c|c|c|c|c|c|c|}
\hline Exposures & Outcomes & SNP selection ${ }^{\mathrm{a}}$ & $\begin{array}{l}\text { Inverse variance weighting } \\
p \text { for sex difference }\end{array}$ & $\begin{array}{l}\text { MR-Egger } \\
p \text { for sex difference }\end{array}$ & $\begin{array}{l}\text { Weighted median } \\
p \text { for sex difference }\end{array}$ & $\begin{array}{l}\text { MR-PRESSO } \\
p \text { for sex difference }\end{array}$ \\
\hline \multicolumn{7}{|c|}{ Effect of lean mass on diabetes and glycaemic traits } \\
\hline \multirow[t]{18}{*}{ Lean mass } & \multirow[t]{3}{*}{ Diabetes } & All & 0.84 & 0.53 & 0.77 & 0.87 \\
\hline & & Lenient & 0.50 & 0.03 & 0.05 & 0.37 \\
\hline & & Strict & 0.47 & 0.67 & 0.01 & 0.93 \\
\hline & \multirow[t]{3}{*}{$\mathrm{HbA}_{1 \mathrm{c}}$} & All & 0.20 & 0.10 & 0.56 & 0.05 \\
\hline & & Lenient & 0.37 & 0.004 & 0.35 & \\
\hline & & Strict & 0.81 & 0.20 & 0.88 & \\
\hline & \multirow[t]{3}{*}{ Fasting glucose } & All & 0.51 & 0.08 & 0.52 & 0.76 \\
\hline & & Lenient & 0.71 & 0.16 & 0.61 & \\
\hline & & Strict & 0.44 & 0.94 & 0.56 & \\
\hline & \multirow[t]{3}{*}{ Fasting insulin } & All & 0.26 & 0.78 & 0.33 & \\
\hline & & Lenient & 0.93 & 0.10 & 0.70 & \\
\hline & & Strict & 0.78 & 0.30 & 1.00 & \\
\hline & \multirow[t]{3}{*}{ HOMA-B } & All & 0.79 & 0.82 & 0.64 & \\
\hline & & Lenient & 0.40 & 0.51 & 0.68 & \\
\hline & & Strict & 0.48 & 0.05 & 0.80 & \\
\hline & \multirow[t]{3}{*}{ HOMA-IR } & All & 0.28 & 0.004 & 0.33 & \\
\hline & & Lenient & 0.12 & 0.002 & 0.74 & \\
\hline & & Strict & 0.23 & 0.05 & 0.16 & \\
\hline \multicolumn{7}{|c|}{ Effect of diabetes and glycaemic traits on lean mass } \\
\hline \multirow[t]{2}{*}{ Diabetes } & \multirow[t]{2}{*}{ Lean mass } & All & 0.53 & 0.69 & 0.27 & 0.06 \\
\hline & & Removed & 0.03 & 0.09 & 0.61 & 0.32 \\
\hline \multirow[t]{2}{*}{$\mathrm{HbA}_{1 \mathrm{c}}$} & & All & 0.66 & 0.99 & 0.29 & 0.36 \\
\hline & & Removed & 0.25 & 0.58 & 0.29 & 0.22 \\
\hline \multirow[t]{2}{*}{ Fasting glucose } & & All & 0.55 & 0.88 & 0.73 & 0.71 \\
\hline & & Removed & 0.08 & 0.90 & 0.08 & 0.03 \\
\hline \multirow[t]{2}{*}{ Fasting insulin } & & All & 0.73 & 0.93 & 0.96 & 0.02 \\
\hline & & Removed & 0.84 & & & \\
\hline HOMA-B & & All & 0.48 & 0.07 & 0.45 & 0.35 \\
\hline
\end{tabular}

MR-PRESSO analysis was only carried out for associations with a significant global test $p$ value $(p<0.05)$ in Table 1 . Associations without an MRPRESSO estimate were not tested for differences by sex

Bonferroni corrected significance level $(0.05 / 41=0.001)$ was used to correct for multiple comparisons

a 'All' represents analyses without removing pleiotropic SNPs; 'Lenient' represents analyses after removing pleiotropic SNPs using a lenient approach; 'Strict' represents analyses after removing pleiotropic SNPs using a strict approach; 'Removed' represents analyses after removing pleiotropic SNPs

pleiotropy definition and MR-PRESSO, but not using the strict definition although the estimate was similar. $I^{2}$ was lower after removing pleiotropic SNPs (Table 1).

\section{Influence of genetically predicted diabetes risk and glycaemic traits on lean mass and grip strength}

We obtained 141 SNPs for diabetes, 35 for $\mathrm{HbA}_{1 \mathrm{c}}, 30$ for fasting glucose, 14 for fasting insulin and five for HOMA$\mathrm{B}$, after removing correlated SNPs. HOMA-IR was excluded from the analyses because there were no genome-wide significant SNPs. All the above SNPs had $F$ statistics larger than 10 with overall $F$ statistics of 70.8 for diabetes, 154.9 for $\mathrm{HbA}_{1 \mathrm{c}}, 118.1$ for fasting glucose, 42.6 for fasting insulin and 46.4 for HOMA-B. Attributes of these SNPs are shown in ESM Table 5. Among the included SNPs, 68, 12,14 and 12 SNPs were considered to be pleiotropic SNPs for diabetes, $\mathrm{HbA}_{1 \mathrm{c}}$, fasting glucose and fasting insulin, respectively, as they may be directly or indirectly related to the outcomes (lean mass/grip strength) after searching on Phenoscanner and/or the GWAS Catalog. No pleiotropic SNPs were identified for HOMA-B. 


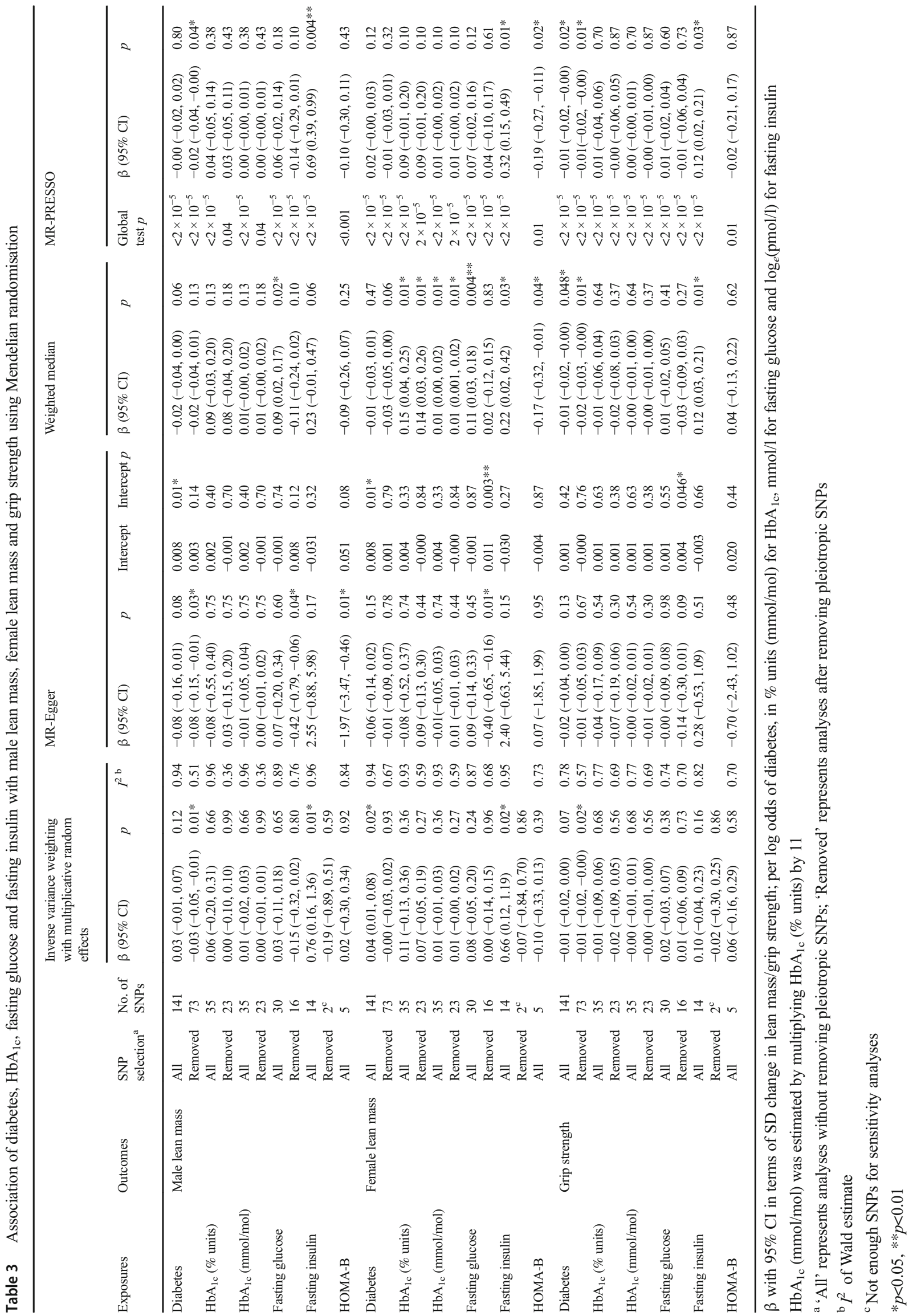


ESM Table 6 shows the phenotypes related to the potentially pleiotropic SNPs.

Table 3 shows that diabetes was inversely and significantly associated with male lean mass and grip strength after removing pleiotropic SNPs as well as being robust to sensitivity analysis using MR-PRESSO. $\mathrm{HbA}_{1 \mathrm{c}}$, fasting glucose and HOMA-B were not significantly associated with grip strength in any analyses or to lean mass in most analyses, especially after removing pleiotropic SNPs or outliers. Fasting insulin was positively and significantly associated with male and female lean mass and possibly with grip strength before removing pleiotropic SNPs but no significant association was found after removing pleiotropic SNPs. No significant difference by sex was found.

Power calculations showed at least $80 \%$ power to detect an OR of 0.94 and 0.81 per 1 SD of lean mass and grip strength, respectively. Results from power calculations are shown in ESM Table 7.

\section{Discussion}

Consistent with the randomised controlled trials in people with metabolic diseases [6], we found some evidence that grip strength (an indicator of muscle strength) and lean mass could be associated with diabetes in the general population. Conversely, diabetes could be associated with lower whole body lean mass and lower grip strength while glycaemic traits generally showed no association. To our knowledge, this is the first Mendelian randomisation study using the most recent GWAS to assess the bi-directional relations between muscle mass and grip strength on the one hand and diabetes and glycaemic traits on the other hand.

Our results suggest that female lean mass was related to diabetes while male lean mass was not, although the estimates were directionally similar with no strong evidence of sexspecific effects. We also cannot rule out that grip strength may be inversely associated with diabetes consistent with previous studies on grip strength [11, 39]. An inverse association of diabetes with lean mass and grip strength is consistent with previous studies suggesting that diabetes may directly affect skeletal muscle [40, 41]. By contrast, fasting insulin also showed a possible positive association with lean mass, consistent with its role as a growth promoter. However, given the lack of robustness to sensitivity analyses this result requires replication.

A possible mechanism for the inverse association of grip strength (and possibly lean mass) with diabetes could be an increase in insulin action and lowering of blood glucose among people with higher muscle strength, which may help to reduce the risk of diabetes $[42,43]$. However, lean mass and grip strength were not shown to benefit glycaemic traits in our analyses. Further investigation of the effect of muscle strength on postprandial glucose and insulin activity could provide more insight into any possible mechanism. The inverse association may also be a possible cause of the low obesity but high diabetes rate in Asian populations compared with Western populations, as Asian individuals usually have a lower muscle mass, thus lower muscle strength [44, 45].

The association of lower muscle mass with diabetes has also been discussed in previous studies where the optimal form of exercise for reducing diabetes is in question [46]. Our results may suggest increasing both muscle mass and strength could be associated with lower risk of diabetes. Therefore, promotion of both muscle mass and strength building may be possible interventions for reducing diabetes risk.

The choice of genetic predictors is also an important point to note. In contrast to a recent study that assessed the effect of grip strength on frailty indices [18], we have chosen absolute, rather than relative, grip strength because relative grip strength (grip strength/body weight) takes account of weight, which represents lean mass, bone mass and fat mass, making the estimates more a measure of overall muscle fitness than muscle strength alone. Moreover, a change in relative grip strength may not only represent a change in grip strength but also a change in body fat mass. As such, absolute grip strength may be a better proxy for examining the effect of muscle strength alone.

Although we used a bi-directional Mendelian randomisation design, which is more resistant to confounding, and used publicly available GWAS data to reduce the likelihood of underpowered analyses, there are some limitations, primarily concerning the assumptions of Mendelian randomisation. First, the SNPs are assumed to be strongly associated with the exposure, as indicated by a high $F$ statistic and SNPs reaching genome-wide significance in the most recent GWAS. Second, the SNPs are assumed to be independent of confounders of the exposure-outcome relation, as has been empirically shown elsewhere [47]. Third, the SNPs are assumed to affect the outcomes through the exposure of interest only, i.e. the exclusion restriction assumption. To identify these potential pleiotropic effects, we checked for associated phenotypes of the SNPs using both the GWAS Catalog and Phenoscanner, and we obtained estimates without these potentially invalid SNPs. In addition, it is difficult to determine all pleiotropic SNPs as the complex relationship between some phenotypes might not be well understood; therefore the results should be interpreted with caution. Specifically, it is unclear which aspects of body size and composition, such as height, act on diabetes via mechanisms other than fat mass, muscle mass and muscle quality, i.e. grip strength. In this way, strictly removing genetic variants because of potential pleiotropy may also have removed a true effect. Fourth, population stratification might affect the estimates. However, the SNP-exposure and SNP-outcome association were mainly obtained from European individuals with genomic control, which should have minimised this possibility. 
Fifth, our results were mainly based on people of European ancestry and may not extend to other populations. However, we would expect the causal effects of grip strength and lean mass to be similar across all populations, although they might be more relevant in some specific populations. Sixth, we cannot determine the clinical significance of the results because Mendelian randomisation should be interpreted as testing for causation rather than indicating the size of any causal effect. Although a small effect size may not be clinically significant, the effect on population health may still be important. Seventh, the effect of SNPs on phenotype may be compensated for during development, i.e. canalisation [7]; however, whether such canalisation effects exist is unknown. Eighth, our study may be inadequately powered to detect differences by sex. Finally, we rely on the integrity of the underlying GWAS, particularly those based on case-control studies, whose design may not include consideration of selection bias by prior death [48, 49]. Such selection bias is most likely to occur for exposures that cause premature death and diseases that occur at older ages, and so may not be particularly relevant here.

\section{Conclusion}

Our study suggests that muscle mass and strength may have some favourable effects on diabetes. Conversely, diabetes may be related to reduced muscle mass and grip strength. Because muscle building has the advantage of being a potentially attractive target, interventions on muscle strength building, such as resistance training, could be further investigated as possible diabetes-preventive measures.

Acknowledgements Data on diabetes have been contributed by DIAGRAM investigators and have been downloaded from http://www. diagram-consortium.org. Data on glycaemic traits have been contributed by MAGIC investigators and have been downloaded from www. magicinvestigators.org.

Data availability All data are available in the submitted manuscript, supplementary files or related sources described in the manuscript.

Funding This research received no specific grant from any funding agency in the public, commercial or not-for-profit sectors.

Duality of interest The authors declare that there is no duality of interest associated with this manuscript.

Contribution statement $\mathrm{CHCY}$ conducted the literature review and the analysis and drafted the manuscript. SLAY, CMS and SSMF conceptualised ideas and designed the study. SLAY and CMS directed the analytical strategy and supervised the study from conception to completion. SLAY, CMS and SSMF revised drafts of the manuscript. All the authors contributed to the interpretation of the data, critically revising the paper and approval of the final version. CHCY is the guarantor of this work.

Publisher's note Springer Nature remains neutral with regard to jurisdictional claims in published maps and institutional affiliations.

\section{References}

1. Yoon KH, Lee JH, Kim JW et al (2006) Epidemic obesity and type 2 diabetes in Asia. Lancet (London, England) 368(9548):16811688. https://doi.org/10.1016/s0140-6736(06)69703-1

2. Wang T, Huang T, Li Y et al (2016) Low birthweight and risk of type 2 diabetes: a Mendelian randomisation study. Diabetologia 59(9):1920-1927. https://doi.org/10.1007/s00125-016-4019-z

3. Bann D, Wills A, Cooper R et al (2014) Birth weight and growth from infancy to late adolescence in relation to fat and lean mass in early old age: findings from the MRC National Survey of Health and Development. Int J Obes 38(1):69-75. https://doi.org/10.1038/ ijo.2013.115

4. Smith AG, Muscat GEO (2005) Skeletal muscle and nuclear hormone receptors: implications for cardiovascular and metabolic disease. Int J Biochem Cell Biol 37(10):2047-2063. https://doi.org/10. 1016/j.biocel.2005.03.002

5. Schooling CM, Jiang C, Zhang W, Lam TH, Cheng KK, Leung GM (2011) Adolescent build and diabetes: the Guangzhou Biobank Cohort Study. Ann Epidemiol 21(1):61-66. https://doi.org/10. 1016/j.annepidem.2010.08.010

6. Strasser B, Siebert U, Schobersberger W (2010) Resistance training in the treatment of the metabolic syndrome: a systematic review and meta-analysis of the effect of resistance training on metabolic clustering in patients with abnormal glucose metabolism. Sports Med (Auckland, NZ) 40(5):397-415. https://doi.org/10.2165/11531380000000000-00000

7. Lawlor DA, Harbord RM, Sterne JA, Timpson N, Davey Smith G (2008) Mendelian randomization: using genes as instruments for making causal inferences in epidemiology. Stat Med 27(8):11331163. https://doi.org/10.1002/sim.3034

8. Zheng J, Baird D, Borges MC et al (2017) Recent developments in Mendelian randomization studies. Curr Epidemiol Rep 4(4):330 345. https://doi.org/10.1007/s40471-017-0128-6

9. Lawlor DA (2016) Commentary: Two-sample Mendelian randomization: opportunities and challenges. Int J Epidemiol 45(3):908915. https://doi.org/10.1093/ije/dyw127

10. Taylor AE, Davies NM, Ware JJ, VanderWeele T, Smith GD, Munafo MR (2014) Mendelian randomization in health research: using appropriate genetic variants and avoiding biased estimates. Econ Hum Biol 13:99-106. https://doi.org/10.1016/j.ehb.2013.12.002

11. Xu L, Hao YT (2017) Effect of handgrip on coronary artery disease and myocardial infarction: a Mendelian randomization study. Sci Rep 7(1):954. https://doi.org/10.1038/s41598-017-01073-z

12. The Neale Lab (2018) GWAS results. Available from http://www. nealelab.is/uk-biobank/, accessed 28 Oct 2018

13. Sudlow C, Gallacher J, Allen N et al (2015) UK Biobank: an open access resource for identifying the causes of a wide range of complex diseases of middle and old age. PLoS Med 12(3):e1001779. https://doi.org/10.1371/journal.pmed.1001779

14. UK Biobank (2011) Grip strength measurement. Available from http://biobank.ctsu.ox.ac.uk/crystal/docs/Gripstrength.pdf, accessed 28 Oct 2018

15. Howrigan D (2017) Details and considerations of the UK Biobank GWAS. Available from http://www.nealelab.is/blog/2017/9/11/ details-and-considerations-of-the-uk-biobank-gwas, accessed 28 Oct 2018

16. Zillikens MC, Demissie S, Hsu YH et al (2017) Large metaanalysis of genome-wide association studies identifies five loci for lean body mass. Nat Commun 8(1):80. https://doi.org/10. 1038/s41467-017-00031-7

17. Visser M, Fuerst T, Lang T, Salamone L, Harris TB (1999) Validity of fan-beam dual-energy X-ray absorptiometry for measuring fatfree mass and leg muscle mass. Health, Aging, and Body Composition Study-Dual-Energy X-ray Absorptiometry and 
Body Composition Working Group. J Appl Physiol 87(4):15131520. https://doi.org/10.1152/jappl.1999.87.4.1513

18. Tikkanen E, Gustafsson S, Amar D et al (2018) Biological insights into muscular strength: genetic findings in the UK Biobank. Sci Rep 8(1):6451. https://doi.org/10.1038/s41598-018-24735-y

19. Wind AE, Takken T, Helders PJ, Engelbert RH (2010) Is grip strength a predictor for total muscle strength in healthy children, adolescents, and young adults? Eur J Pediatr 169(3):281-287. https://doi.org/10.1007/s00431-009-1010-4

20. Mahajan A, Taliun D, Thurner M et al (2018) Fine-mapping type 2 diabetes loci to single-variant resolution using high-density imputation and islet-specific epigenome maps. Nat Genet 50(11):15051513. https://doi.org/10.1038/s41588-018-0241-6

21. Wheeler E, Leong A, Liu C-T et al (2017) Impact of common genetic determinants of hemoglobin A1c on type 2 diabetes risk and diagnosis in ancestrally diverse populations: a transethnic genome-wide meta-analysis. PLoS Med 14(9):e1002383. https:// doi.org/10.1371/journal.pmed.1002383

22. Dupuis J, Langenberg C, Prokopenko I et al (2010) New genetic loci implicated in fasting glucose homeostasis and their impact on type 2 diabetes risk. Nat Genet 42(2):105-116. https://doi.org/10.1038/ng.520

23. Manning AK, Hivert M-F, Scott RA et al (2012) A genome-wide approach accounting for body mass index identifies genetic variants influencing fasting glycemic traits and insulin resistance. Nat Genet 44(6):659-669. https://doi.org/10.1038/ng.2274

24. Matthews DR, Hosker JP, Rudenski AS, Naylor BA, Treacher DF, Turner RC (1985) Homeostasis model assessment: insulin resistance and beta-cell function from fasting plasma glucose and insulin concentrations in man. Diabetologia 28(7):412-419. https://doi.org/ 10.1007/BF00280883

25. Scott RA, Lagou V, Welch RP et al (2012) Large-scale association analyses identify new loci influencing glycemic traits and provide insight into the underlying biological pathways. Nat Genet 44(9): 991-1005. https://doi.org/10.1038/ng.2385

26. Pierce BL, Ahsan H, VanderWeele TJ (2011) Power and instrument strength requirements for Mendelian randomization studies using multiple genetic variants. Int J Epidemiol 40(3):740-752. https:// doi.org/10.1093/ije/dyq151

27. Bowden J, Del Greco MF, Minelli C, Davey Smith G, Sheehan NA, Thompson JR (2016) Assessing the suitability of summary data for two-sample Mendelian randomization analyses using MR-Egger regression: the role of the $I^{2}$ statistic. Int J Epidemiol 45(6):19611974. https://doi.org/10.1093/ije/dyw220

28. Burgess S (2014) Sample size and power calculations in Mendelian randomization with a single instrumental variable and a binary outcome. Int J Epidemiol 43(3):922-929. https://doi.org/10.1093/ije/ dyu005

29. Staley JR, Blackshaw J, Kamat MA et al (2016) PhenoScanner: a database of human genotype-phenotype associations. Bioinformatics (Oxford, England) 32(20):3207-3209. https://doi. org/10.1093/bioinformatics/btw373

30. MacArthur J, Bowler E, Cerezo M et al (2017) The new NHGRIEBI Catalog of published genome-wide association studies (GWAS Catalog). Nucleic Acids Res 45(D1):D896-D901. https://doi.org/ 10.1093/nar/gkw1133

31. Verbanck M, Chen C-Y, Neale B, Do R (2018) Detection of widespread horizontal pleiotropy in causal relationships inferred from Mendelian randomization between complex traits and diseases. Nat Genet 50(5):693-698. https://doi.org/10.1038/s41588-018-0099-7

32. Brion M-JA, Shakhbazov K, Visscher PM (2013) Calculating statistical power in Mendelian randomization studies. Int J Epidemiol 42(5):1497-1501. https://doi.org/10.1093/ije/dyt179

33. Robinson C, Tomek S, Schumacker R (2013) Tests of moderation effects: difference in simple slopes versus the interaction term. Multiple Linear Regression Viewpoints 39:16-25
34. Hemani G, Zheng J, Elsworth B et al (2018) The MR-Base platform supports systematic causal inference across the human phenome. eLife 7:e34408. https://doi.org/10.7554/eLife.34408

35. R Core Team (2016) R: A language and environment for statistical computing. R Foundation for Statistical Computing, Vienna, Austria

36. Bowden J, Davey Smith G, Haycock PC, Burgess S (2016) Consistent estimation in Mendelian randomization with some invalid instruments using a weighted median estimator. Genet Epidemiol 40(4):304-314. https://doi.org/10.1002/gepi.21965

37. Bowden J, Davey Smith G, Burgess S (2015) Mendelian randomization with invalid instruments: effect estimation and bias detection through Egger regression. Int J Epidemiol 44(2):512-525. https://doi.org/10.1093/ije/dyv080

38. Gomez-Ambrosi J, Silva C, Galofre JC et al (2011) Body adiposity and type 2 diabetes: increased risk with a high body fat percentage even having a normal BMI. Obesity 19(7):1439-1444. https://doi. org/10.1038/oby.2011.36

39. Li JJ, Wittert GA, Vincent A et al (2016) Muscle grip strength predicts incident type 2 diabetes: population-based cohort study. Metabolism 65(6):883-892. https://doi.org/10.1016/j.metabol. 2016.03.011

40. Kalyani RR, Corriere M, Ferrucci L (2014) Age-related and disease-related muscle loss: the effect of diabetes, obesity, and other diseases. Lancet Diabetes Endocrinol 2(10):819-829. https://doi. org/10.1016/s2213-8587(14)70034-8

41. Kalyani RR, Saudek CD, Brancati FL, Selvin E (2010) Association of diabetes, comorbidities, and $\mathrm{A} 1 \mathrm{C}$ with functional disability in older adults: results from the National Health and Nutrition Examination Survey (NHANES), 1999-2006. Diabetes Care 33(5):1055-1060. https://doi.org/10.2337/dc09-1597

42. Holten MK, Zacho M, Gaster M, Juel C et al (2004) Strength training increases insulin-mediated glucose uptake, GLUT4 content, and insulin signaling in skeletal muscle in patients with type 2 diabetes. Diabetes 53(2):294-305. https://doi.org/10.2337/ diabetes.53.2.294

43. Geirsdottir OG, Arnarson A, Briem K, Ramel A, Jonsson PV, Thorsdottir I (2012) Effect of 12-week resistance exercise program on body composition, muscle strength, physical function, and glucose metabolism in healthy, insulin-resistant, and diabetic elderly Icelanders. J Gerontol A Biol Sci Med Sci 67(11):1259-1265. https://doi.org/10.1093/gerona/gls096

44. Ramachandran A, Wan Ma RC, Snehalatha C (2010) Diabetes in Asia. Lancet 375(9712):408-418. https://doi.org/10.1016/S01406736(09)60937-5

45. Rush EC, Freitas I, Plank LD (2009) Body size, body composition and fat distribution: comparative analysis of European, Maori, Pacific Island and Asian Indian adults. Br J Nutr 102(04):632641. https://doi.org/10.1017/S0007114508207221

46. Hou WW, Tse MA, Lam TH, Leung GM, Schooling CM (2015) Adolescent testosterone, muscle mass and glucose metabolism: evidence from the 'Children of 1997' birth cohort in Hong Kong. Diabet Med 32(4):505-512. https://doi.org/10.1111/dme.12602

47. Davey Smith G, Lawlor DA, Harbord R, Timpson N, Day I, Ebrahim S (2007) Clustered environments and randomized genes: a fundamental distinction between conventional and genetic epidemiology. PLoS Med 4(12):e352. https://doi.org/10.1371/journal. pmed.0040352

48. Schooling CM (2018) Selection bias in population-representative studies? A commentary on Deaton and Cartwright. Soc Sci Med. https://doi.org/10.1016/j.socscimed.2018.04.047

49. Anderson CD, Nalls MA, Biffi A et al (2011) The effect of survival bias on case-control genetic association studies of highly lethal diseases. Circ Cardiovasc Genet 4(2):188-196. https://doi.org/10. 1161/CIRCGENETICS.110.957928 